\title{
CARACTÉristiques des POPUlations de OSTERTAGia sp. chez les Ovins naturellement infestés de la région de Moulay-Bouazza (Maroc).
}

\author{
J. CABARET*
}

RÉSUMÉ. Soixante-dix caillettes de brebis ont été récoltées mensuellement à l'abbattoir de Moulay-Bouazza (Maroc) durant deux ans. Les caractéristiques de la population d'Ostertagia (principalement $O$. circumcincta) ont été étudiées : nombre d'œufs par femelle, polymorphisme de l'appareil supra-vulvaire, longueur, sex-ratio et densité parasitaire.

Ces paramètres sont liés et peuvent rendre compte des variations saisonnières du nombre d'œufs par femelle.

\section{Characteristics of Ostertagia sp. populations harboured by naturally infected sheep of the Moulay-Bouazza region (Moroceo).}

SUMMARY. Sixty-six abomasa of ewes were collected monthly at the slaughter-house of MoulayBouazza (Morocco) during two years. The characteristics of the population of Ostertagia (mostly $O$. circumcincta) were studied : number of eggs per female, vulvar polymorphism, length, sex-ratio and size of the worm-burden.

These parameters were interrelated and could account for the seasonal variations of the number of eggs per female.

Les caractéristiques des populations de Nématodes sont nombreuses. Nous avons retenu celles qui pouvaient présenter un intérêt pour la pathologie (taille et par suite biomasse parasitaire) ou l'épidémiologie. Certains facteurs ont déjà été envisagés : le sex-ratio (Grundmann, Warnock, Wassom, 1976), le polymorphisme de l'appareil supra-vulvaire chez Haemonchus contortus (Lejambre, Whitlock 1968; Lejambre, Ractliffe 1976 ; Lejambre, Whitlock 1976 ; Cabaret 1979). Les études relatives au nombre d'œufs présents dans les femelles de Nématodes sont rares et concernent Trichostrongylus colubriformis (Chiejina et Sewell, 1974) chez les Ovins, Strongylus sp. ou T. axei (Ogbourne, 1971) chez le cheval.

* Institut National de la Recherche Agronomique, Centre de Recherches de Tours-Nouzilly, F 37380 Monnaie.

Accepté le 7 janvier 1983 . 
Les données concernant Ostertagia sp., un des Strongles digestifs dominant de la caillette des moutons, sont très partielles. Une étude saisonnière des caractéristiques citées, objet du présent travail, devrait permettre de mieux comprendre les phénomènes de régulation de cette population.

\section{Matériel et méthodes}

Les prélèvements de caillette ont été réalisés à l'abattoir sur des femelles adultes non gestantes infestées naturellement et originaires des environs de Moulay-Bouazza. Les récoltes ont concerné 66 brebis, au rythme de 3 par mois, en 1978 et 1979.

Les parasites ont été examinés sur une partie aliquote $\left(1 / 5^{\mathrm{e}}\right.$ à $\left.1 / 10^{\mathrm{e}}\right)$ du contenu digestif après tamisage. Les principaux parasites sont : Ostertagia circumcincta, Marshallagia marshalli et Trichostrongylus axei (Cabaret et Ouhelli, 1978 ; Cabaret, 1979). La population des Ostertagia est constitué par une espèce dominante $O$. circumcincta et des espèces accessoires (moins de $5 \%$ des individus : $O$. trifurcata, $O$. ostertagi). 876 femelles d'Ostertagia ont été examinées. Les paramètres mesurés étaient : la taille des femelles, la morphologie supravulvaire, le nombre d'œufs dans l'utérus, la taille des mâles, le nombre total d'Ostertagia dans la caillette.

\section{Résultats}

L'examen des données est envisagé à deux niveaux : celui d'une population vermineuse au sein d'un individu-hôte et celui d'un regroupement de populations de divers hôtes à la dimension du mois ou de la saison.

\section{Données individuelles}

Elles ne concernent que la population des Ostertagia sur trois brebis fortement infestées afin que les calculs de corrélation soient puissants.

Le nombre d'œufs par femelle (O.P.F.) est significativement lié à la taille $\mathrm{t}$ (en $\mathrm{mm})$ :

$$
\begin{array}{rll}
\text { animal } 1 \text { : O.P.F. }=-40,8+5,95 \mathrm{t} & 48 \text { femelles } & \mathrm{r}=0,50 \\
2 \text { : O.P.F. }=-35,9+6,57 \mathrm{t} & 31 \text { femelles } & \mathrm{r}=0,50 \\
3 \text { : O.P.F. }=-74,9+9,63 \mathrm{t} & 25 \text { femelles } & \mathrm{r}=0,69
\end{array}
$$

Pour chacun des individus-hôtes, le nombre d'œufs par femelle est plus faible chez les femelles sans processus supra-vulvaire (lisse) au seuil de signification de $2 \%$. Ceci est peut-être lié au fait que les femelles lisses sont plus petites que les femelles ayant un processus supra-vulvaire (seuil de signification de $4 \%$ ).

\section{Moyennes mensuelles}

Les résultats sont présentés dans le tableau $I$ et les liaisons entre les diverses mesures sur la figure 1. L'influence de la densité vermineuse est exposée dans le 
TABLEAU I. - Caractéristiques mensuelles moyennes de la population d'Ostertagia sp.

\begin{tabular}{|c|c|c|c|c|c|c|}
\hline Année & Mois & O.P.F. & $\% \mathrm{~L}$. & t.F. & t.M. & $\begin{array}{l}\text { Nombre de } \\
\text { nématodes }\end{array}$ \\
\hline 1978 & $\begin{array}{l}\mathrm{J} \\
\mathrm{F} \\
\mathrm{M} \\
\mathrm{A} \\
\mathrm{M} \\
\mathrm{J} \\
\mathrm{J} \\
\mathrm{S} \\
\mathrm{O} \\
\mathrm{N} \\
\mathrm{D}\end{array}$ & $\begin{array}{l}31,0 \\
32,3 \\
27,7 \\
34,9 \\
36,7 \\
20,0 \\
22,7 \\
25,6 \\
31,5 \\
38,2 \\
45,9\end{array}$ & $\begin{array}{r}48,4 \\
27,1 \\
48,0 \\
18,5 \\
41,7 \\
6,7 \\
25,0 \\
11,6 \\
37,7 \\
25,8 \\
11,9\end{array}$ & $\begin{array}{l}10,3 \\
11,8 \\
11,5 \\
12,1 \\
11,1 \\
12,7 \\
10,3 \\
11,6 \\
11,9 \\
12,3 \\
13,0\end{array}$ & $\begin{array}{l}8,9 \\
8,9 \\
8,6 \\
9,2 \\
8,4 \\
9,9 \\
9,4 \\
9,5 \\
8,8 \\
9,1 \\
9,3\end{array}$ & $\begin{array}{r}1326 \\
473 \\
1240 \\
980 \\
420 \\
840 \\
173 \\
1380 \\
1190 \\
373 \\
375\end{array}$ \\
\hline 1979 & $\begin{array}{l}\text { J } \\
\text { F } \\
\text { M } \\
\text { A } \\
\text { M } \\
\text { J } \\
\text { J } \\
\text { S } \\
\text { O } \\
\text { N }\end{array}$ & $\begin{array}{l}49,8 \\
29,6 \\
19,8 \\
36,0 \\
26,7 \\
26,7 \\
27,9 \\
24,6 \\
25,1 \\
34,6\end{array}$ & $\begin{array}{r}17,9 \\
31,6 \\
48,5 \\
28,9 \\
12,5 \\
15,0 \\
8,3 \\
26,5 \\
35,1 \\
61,9\end{array}$ & $\begin{array}{l}13,9 \\
11,3 \\
10,4 \\
11,3 \\
11,2 \\
10,9 \\
11,0 \\
11,0 \\
11,7 \\
11,6\end{array}$ & $\begin{array}{r}10,6 \\
9,2 \\
8,1 \\
9,0 \\
9,2 \\
9,3 \\
8,9 \\
8,9 \\
9,7 \\
9,5\end{array}$ & $\begin{array}{r}250 \\
927 \\
1214 \\
1680 \\
373 \\
327 \\
320 \\
700 \\
494 \\
780\end{array}$ \\
\hline
\end{tabular}

O.P.F. : œuf par femelle.

$\%$ L. : pourcentage de femelles lisses.

t.F. : taille des femelles en $\mathrm{mm}$.

t.M. : taille des mâles d'Ostertagia circumcincta en mm.

TABleau II. - Caractéristiques de la population femelle d'Ostertagia sp. selon la densité vermineuse.

\begin{tabular}{lcccc}
\hline $\begin{array}{c}\text { Densité } \\
\text { vermineuse }\end{array}$ & O.P.F. & $\%$ L. & t.F. & $\begin{array}{c}\text { Proportion de femelles } \\
\text { dans la population } \\
\text { d'Ostertagia }\end{array}$ \\
\hline $\begin{array}{l}<1 \text { ovins) } \\
\left(\begin{array}{l}41 \text { ov } \\
400-1000 \\
(27 \text { ovins })\end{array}\right.\end{array}$ & $34,0 \pm 4,0$ & $16,6 \pm 2,5$ & $11,8 \pm 1,0$ & $0,739 \pm 0,08$ \\
$\begin{array}{l}>1 \text { 000 } \\
(18 \text { ovins })\end{array}$ & $28,6 \pm 2,1$ & $37,2 \pm 5,8$ & $11,2 \pm 0,5$ & $0,634 \pm 0,03$ \\
\hline
\end{tabular}

O.P.F. : œuf par femelle.

$\%$ L. : pourcentage de femelles lisses.

t.F. : taille des femelles (en mm). 
tableau II ; lorsque la densité augmente, le pourcentage de femelles lisses s'accroît tandis que le pourcentage de femelles dans la population vermineuse et le nombre d'œufs par femelle décroissent significativement.

\section{Variations des paramètres dans le temps}

Ceux-ci fluctuent peu d'une année sur l'autre : la variation relative est de 0 à $1,7 \%$ en ce qui concerne la taille moyenne, le pourcentage de femelles lisses et le nombre d'œufs par femelles, de $4,5 \%$ pour le sex-ratio et de $11,4 \%$ pour la densité vermineuse. La saison sèche (15 mai au 15 octobre), par rapport à la saison humide, présente une population à O.P.F. faible $(24,6 \pm 2,3$ contre $33,2 \pm 4,0)$ et à pourcentage réduit de femelles lisses $(15,5 \pm 3,4$ contre $33,0 \pm 3,8)$.

\section{Discussion}

Les interactions nombreuses entre les facteurs permettent l'obtention d'un équilibre dans les populations d'Ostertagia, tant pour leur taille que pour leur nombre ou leur sex-ratio comme le démontre l'examen des données annuelles. A partir de la figure 1 on peut envisager les mécanismes suivants :

- la densité vermineuse augmente : la proportion de femelles va diminuer, les femelles lisses seront présentes en plus grand nombre. Celles-ci sont de petite taille et hébergent par suite un nombre réduit d'œufs.

- la première conséquence sera donc une réduction relative de l'élimination d'œufs, l'O.P.F. et le nombre d'œufs émis étant liés (Chiejina et Sewell, 1974).

- la seconde consistera en une réduction de la proportion des femelles dans la popu-

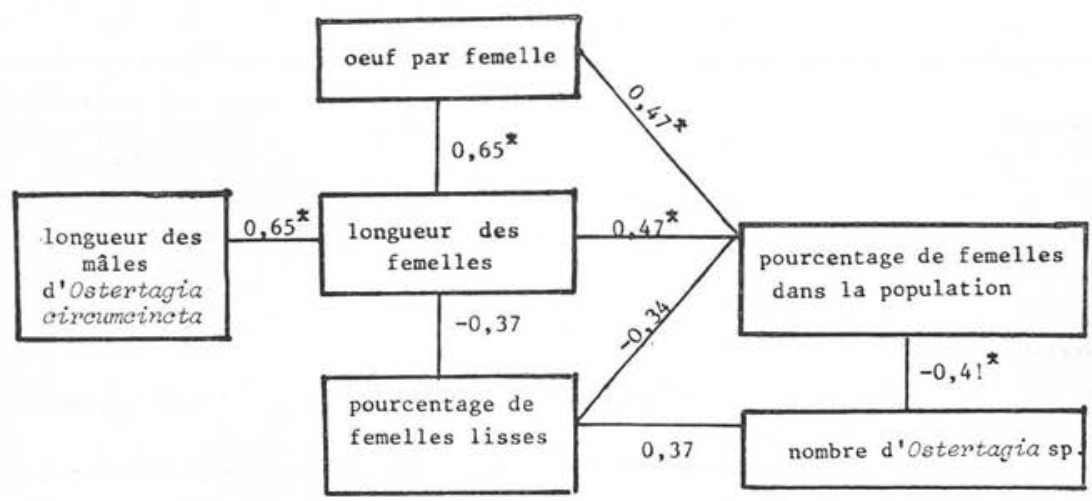

FIG. I. - Relation entre les paramètres de la population d'Ostertagia sp.

* Significatif au seuil de 5 p. cent. 
lation vermineuse, ce qui réduira également la production d'œufs émis dans le milieu extérieur.

- la troisième devrait être la diminution relative de la biomasse parasitaire, les individus étant de petite taille.

Les variations du sex-ratio selon la densité ont été mises en évidence chez les Ankylostomes (Roche et Patrzek, 1966) et celles concernant la taille chez Haemonchus contortus (Ractliffe, Lejambre, Uhazy et Whitlock, 1971) et ne sont pas spécifiques à Ostertagia sp. L'intervention du polymorphisme des femelles a été démontré chez $H$. contortus (Lejambre et Ractliffe, 1976). Pour les ovins de cette étude, nous avons également observé l'existence de différences entre les morphes linguiformes (taille $15,4 \pm 0,5 \mathrm{~mm}$ et O.P.F. $25,6 \pm 1,6$ ) et lisses (tailles $14,3 \pm 0,5$ et O.P.F. $17,3 \pm 1,4$ ) de Marshallagia marshalli.

Des variations saisonnières de fécondité ont été enregistrées par Obgourne (1971) pour Strongylus vulgaris et Trichostrongylus axei chez le cheval. Des fluctuations du nombre d'œufs par femelle sont également observées chez Ostertagia : cet indice est particulièrement faible au cours de la saison sèche. D'autres travaux (Pandey, Cabaret, Ouhelli et Dakkak, 1980) montrent que l'acquisition d'une nouvelle infestation est nulle en saison sèche et que deux pics d'infestation vermineuse sont présents (fin d'hiver et début d'automne). La période sèche correspond donc à une population vieillissante, avec une densité vermineuse faible, une proportion de femelles importante, un nombre d'œufs par femelle très inférieur à celui de la saison humide. Ces résultats sont en accord avec ceux d'autres auteurs (Michel, Lancaster, Hong, 1978 ; Chiejina et Sewell, 1974) lorsque l'on considère que le facteur essentiel est la densité parasitaire. Toutefois la proportion des femelles lisses évolue de façon différente pour Michel, Lancaster et Hong (1978) lors d'infestations expérimentales de bovins par O. ostertagia : les hautes densités sont associées à un pourcentage faible de femelles lisses.

L'étude des caractéristiques des populations d'Ostertagia sp. permet de mieux comprendre les modalités d'atteinte d'un équilibre au niveau annuel. Les paramètres étant interactifs, un travail expérimental permettrait de mieux préciser l'incidence de chacun d'entre eux.

\section{BIBLIOGRAPHIE}

CABARET J. : Variations qualitatives et quantitatives des populations d'Haemonchus contortus chez les Ovins dans deux régions du Maroc. Rev. Med. Vet., 1979, 130, ror 7-1025.

Cabaret J., Ouhelli H. : Étude d'une population de nématodes parasites du tube digestif des Ovins dans la région de Moulay-Bouazza (Moyen-Atlas du Maroc). Rev. Med. Vet., I978, I29, 6о3-6ro.

Chiejina S. N., Sewell M. H. : Experimental infections with Trichostrongylus colubriformis in lambs. Worm burden, growth rate and host-resistance resulting from prolonged escalating infections. Parasitology, 1974, 69, 30I-314.

Grundmann A. W., Warnock R. G., WASSOM D. L. : Some mechanisms of natural regulation of parasitic helminth populations. The Am. Mid. Naturalist, 1976, 95, 347-360.

LejAmbre L. F., Whirlock J. H. : Seasonal fluctuations in linguiform morphs of Haemonchus contortus cayugensis. J. Parasitol., 1968, s4, 827-830.

LeJAmbre L. F., WhitLock J. H. : Changes in the hatching rate of Haemonchus contortus eggs between geographic regions. Parasitology, $1976,73,223^{-2} 3^{8}$. 
LEJAMBre L. F., RActlifFE L. H. : Response of Haemonchus contortus cayugensis to a change in the ratio of smooth to linguiform. Parasitology, 1976, 73, 213-222.

Michel J. F., Lancaster M. B., Hong C. : The length of Ostertagia ostertagi in populations of uniform age. Int. J. Parasitol., 1978, 8, 437-441.

Ogbourne C. P. : Variations in the fecundity of Strongylid worms of the horse. Parasitology, r971, $63,289-298$.

Pandey V. S., Cabaret J., Ouhelli H., Dakkak A. : Étude des Nématodes parasites du tube digestif des Ovins adultes dans deux régions du Maroc. Bull. Off. Int. Epizooties, 1980, 92, I35I-I 356 .

Ractliffe L. H., Lejambre L. F., Uhazy L. S., Whitlock J. H. : Density-dependance of the weigth of Haemonchus contortus adults. Int. J. Parasitol., 1971, I, 297-301.

Roche M., PAtrzek D. : The female to male ration (F.M.R.) in hookworms. J. Parasitol., I97I, $\int 2,117-121$. 